\title{
Synthesis and Antimalarial Activity of Lawsone Mannich Base Derivatives
}

\author{
Medhi Arundhati, Chetia Dipak, Rudrapal Mithun* \\ Department of Pharmaceutical Sciences, Dibrugarh University, Dibrugarh, INDIA.
}

\begin{abstract}
Introduction: The emergence of multi-drug resistant strains of Plasmodium falciparum has increasingly become a serious health problem worldwide. To address this challenging issue, there is an urgent need to discover and develop novel and potent antimalarial agents. Materials and Methods: A new series of lawsone Mannich base derivatives were synthesized, characterized (IR, NMR and Mass) and evaluated for in vitro for antimalarial activity against chloroquine (CQ)-sensitive and CQ-resistant strains of $P$. falciparum. Results and Discussion: Among synthesized compounds, five compounds showed good antimalarial activity against both chloroquine (CQ)-sensitive (3D-7) and -resistant (RKL2) strains of $P$. falciparum, which, however, was considerably less than that of the standard reference drug, $\mathrm{CQ}$. The antimalarial activity of these five compounds was found better against sensitive $\left(\mathrm{IC}_{50}=0.411-0.502 \mu \mathrm{g} / \mathrm{ml}\right.$ ) strain than the resistant $\left(\mathrm{IC}_{50}\right.$ $=1.391-2.394 \mu \mathrm{g} / \mathrm{ml}$ ) one. The IC50 values for CO were $0.044 \mu \mathrm{g} / \mathrm{ml}$ and $0.216 \mu \mathrm{g} / \mathrm{ml}$ against sensitive and resistant strains of $P$. falciparum, respectively. SAR study suggests that antimalarial potential of lawsone structural scaffold can be modulated by different substitution pattern like Mannich base substitution (alkyl/aryl/heteroaryl substituted aminoalkyl moiety) at the C-3 position of 1,4-naphthoquinone ring system. Conclusion: Owing to their antimalarial effectiveness, lawsone derived aminonaphthoquinones can be used as lead structure(s) in the development of yet more potent antimalarial agents.
\end{abstract}

Key words: Lawsone, Mannich bases, Aminonaphthoquinone, Plasmodium falciparum, Resistant malaria.

\section{INTRODUCTION}

Malaria is a lethal infectious disease which continues to be a major problem to public health worldwide, affecting hundreds of millions of infections and millions of deaths annually. ${ }^{1,2}$ Plasmodium falciparum is the most deadly species of malaria parasites which causes potentially fatal malaria like cerebral malaria and is therefore responsible for most of the malaria-related deaths around the globe. In patients with severe malaria, the mortality rate is accounted for $20-50 \%$ when left untreated. ${ }^{2,3,4}$ P. falciparum also produces rapid development of drug resistance. In recent years, the emergence of resistant strains of $P$. falciparum against currently available antimalarial drugs has increasingly become a serious threat to prevention and control of malaria. ${ }^{2,5,6}$ Because of this prob- lem, synthetic quinoline antimalarials like chloroquine and mefloquine have become clinically less effective or ineffective in the treatment of resistant $P$. falciparum malaria., ${ }^{2,7}$ Artemisinin based combination therapies (ACTs) [example, artemether+lumefantrin $\mathrm{e}+$ amodiaquine and artesunate + mefloqui ne+piperaquine] are therefore the recommended therapies (WHO, 2015) in multidrug-resistant $P$. falciparum malaria. But, emergence of resistant strains and pharmacokinetic issues have limited the clinical utility of ACTs in the treatment of $P$. falciparum malaria. ${ }^{2,48}$ The discovery and development of potent antimalarial drug molecules is therefore urgently required to address the above challenging issue, particularly, to fight against resistant $P$. falciparum malaria.
Submission Date: 09-09-2017; Revision Date: 08-11-2017; Accepted Date: 09-11-2017

DOI: 10.5530/ijper.52.3.55 Correspondence: Mithun Rudrapal, Department of Pharmaceutical Sciences,

Dibrugarh University,

Dibrugarh-786004,

Assam, INDIA.

Phone: +91-9085726851

E-mail: rudrapal.m03@gmail. com

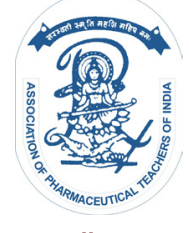

www.ijper.org 


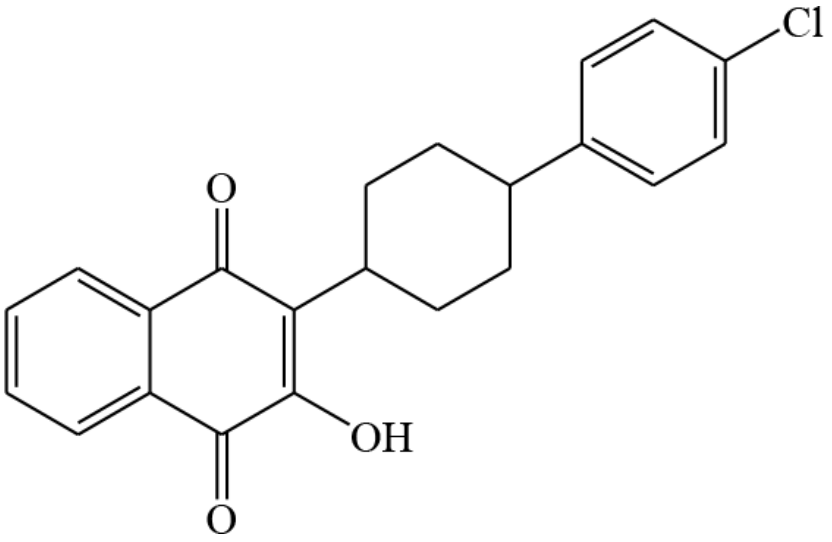

Figure 1: Structure atovaquone (ATQ).

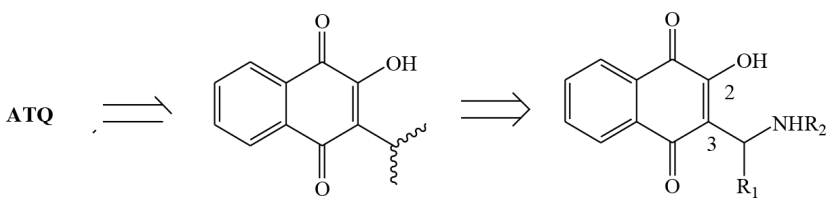

Figure 2: Design of lawsone semi-synthetic analogues (aminonaphthoquinones) as Mannich bases

There is an increasing interest among medicinal chemists towards natural/synthetic sources for the discovery of novel structural lead/scaffolds for antimalarial drug development. Lawsone could serve such a good natural lead for the development of new antimalarial drug molecules. Lawsone, the chief active principle of Lawsonia inermis (Henna), is chemically 2-hydroxy derivative of 1,4-naphthoquinone. The biological potential of Henna leaf extracts is mainly attributed due to lawsone and its natural analogues i.e., other naphthoquinones. Besides, semi-synthetic and synthetic analogues of lawsone have also been reported to possess biological properties such as antibacterial, antifungal, antimalarial, anti-inflammatory, antitubercular, and antioxidant. ${ }^{9,10}$ Atovaquone (ATQ, Figure 1), a 3-substituted derivative of lawsone is a clinically useful antimalarial agent, but it has less clinical efficacy when used alone. ATQ is therefore used in combination with other antimalarial drugs like proguanil for the prophylaxis of $P$. falciparum malaria. The antiparasitic activity of quinones is brought about by several biochemical mechanisms such as competitive inhibition of the cytochrome bc1 complex, generation of reactive oxygen species (ROS), enzymatic inhibition (glutathione reductase), and alkylation. ${ }^{5,11,12}$ Literature reveal that lawsone and its synthetic derivatives especially those containing nitrogen have promising potential as antimalarial, anticancer and antivitral agents. Mannich bases of 2-hydroxy-1,4-naphthoquinone (3-substituted aminoalkyl lawsone) have been reported to exhibit antimalarial activity. ${ }^{10,13,14}$ Chemical modification (Figure 2) of lawsone structural scaffold (residue of ATQ) would therefore be an attractive approach in designing of newer semi-synthetic aminonaphthoquinones as alternative sources of novel antimalarial drug molecules for the treatment of resistant malaria.

In view of above facts, an attempt was made to design and synthesize a series of Mannich base substituted 2-hydroxy-1,4-naphthoquinone analogues as novel semi-synthetic alkyl/aryl/heteroaryl substituted amino derivatives of lawsone for in vitro antimalarial activity evaluation.

\section{MATERIALS AND METHODS \\ Chemicals and Analysis}

All of the chemicals were procured commercially from Sigma-Aldrich Corporation (USA), Merck Specialists Pvt. Ltd. (Germany), HiMedia Lab. Pvt. Ltd. (Germany) or Spectrochem Pvt. Ltd. (India), and were used without further purification. The solvents and reagents used in the antimalarial study were of analytical grade. The progress of reactions was monitored by the silica gel-G thin-layer chromatography (TLC) and the spots were visualized by iodine vapours. Melting points (MP) were measured in open capillaries on an electrically heated melting point apparatus. Ultraviolet (UV)-visible spectra were recorded on Shimadzu UV-1700 UV-visible spectrophotometer and the wave length of maximum absorption $\left(\lambda_{\max }, \mathrm{nm}\right)$ is reported. Infrared (IR) spectra were obtained on a Bruker Alpha FT-IR spectrometer by ATR technique and are reported in terms of frequency of absorption $\left(\mathrm{v}, \mathrm{cm}^{-1}\right) \cdot{ }^{1} \mathrm{H}$ and ${ }^{13} \mathrm{C}$ Nuclear Magnetic Resonance (NMR) spectra were recorded on Bruker Avance II 400 FT-NMR spectrometer at 400 and $100 \mathrm{MHz}$, respectively using TMS as an internal standard $(\delta 0.00 \mathrm{ppm})$ and $\mathrm{CDCl}_{3}$ as a solvent. Chemical shift $(\delta)$ values were expressed in parts per million (ppm) relative to TMS ( $\delta 0.00 \mathrm{ppm}$ ). Mass spectra were obtained with the Waters, Q-TOF Micromass instrument using ESI-MS in the range of $m / z 100-1200$ both in the negative and positive ion modes. The $m / z$ values of the most intense quasimolecular ion $[\mathrm{M}+\mathrm{H}]^{+}$peak, with relative intensities in parentheses are stated.

\section{Synthesis and characterization}

Target compounds, $\mathbf{4 a - j}$ were synthesized according to the method of preparation of Mannich bases from lawsone reported by Sandro et al. ${ }^{14}$ with minor modification. The general procedure of synthesis is outlined below:

A solution of lawsone $(1 \mathrm{mmol})$, respective amine (1.1 $\mathrm{mmol})$ and respective aldehyde $(1.2 \mathrm{mmol})$ was stirred 


\begin{tabular}{|c|c|c|c|c|c|c|c|c|}
\hline \multicolumn{6}{|c|}{ Table 1: Physicochemical details of synthesized lawsone Mannich bases. } \\
\hline Comp. & MF & MW & State & Colour & Yield (\%) & MP ( ${ }^{\circ}$ C) & $R_{f}^{\text {a }}$ & clogP $^{\text {b }}$ \\
\hline $4 \mathrm{a}$ & $\mathrm{C}_{21} \mathrm{H}_{21} \mathrm{~N}_{3} \mathrm{O}_{4}$ & 351.15 & Solid & Orange & 56.6 & $131-134$ & 0.42 & 3.75 \\
\hline $4 \mathrm{~b}$ & $\mathrm{C}_{23} \mathrm{H}_{16} \mathrm{NO}_{3} \mathrm{~F}$ & 373.11 & Solid & Brown & 60.0 & $136-140$ & 0.57 & 4.70 \\
\hline $4 \mathrm{c}$ & $\mathrm{C}_{23} \mathrm{H}_{15} \mathrm{~N}_{3} \mathrm{O}_{7}$ & 445.38 & Solid & Yellow & 70.0 & $165-167$ & 0.62 & 4.38 \\
\hline $4 \mathrm{~d}$ & $\mathrm{C}_{23} \mathrm{H}_{16} \mathrm{~N}_{2} \mathrm{O}_{6}$ & 416.38 & Solid & Orange & 81.0 & $190-193$ & 0.54 & 3.92 \\
\hline $4 \mathrm{e}$ & $\mathrm{C}_{23} \mathrm{H}_{16} \mathrm{~N}_{2} \mathrm{O}_{5}$ & 400.38 & Solid & Orange & 82.5 & $240-243$ & 0.50 & 4.64 \\
\hline $4 \mathrm{f}$ & $\mathrm{C}_{23} \mathrm{H}_{16} \mathrm{~N}_{2} \mathrm{O}_{6}$ & 416.38 & Solid & Orange & 60.0 & $165-168$ & 0.75 & 3.97 \\
\hline $4 \mathrm{~g}$ & $\mathrm{C}_{21} \mathrm{H}_{20} \mathrm{~N}_{2} \mathrm{O}_{3}$ & 348.40 & Solid & Red & 82.0 & $185-187$ & 0.74 & 3.59 \\
\hline $4 \mathrm{~h}$ & $\mathrm{C}_{21} \mathrm{H}_{19} \mathrm{~N}_{3} \mathrm{O}_{5}$ & 393.39 & Solid & Orange & 79.0 & $187-190$ & 0.52 & 3.34 \\
\hline $4 \mathrm{i}$ & $\mathrm{C}_{21} \mathrm{H}_{20} \mathrm{~N}_{2} \mathrm{O}_{4}$ & 364.39 & Solid & Red & 80.0 & $178-182$ & 0.72 & 2.88 \\
\hline $4 \mathrm{j}$ & $\mathrm{C}_{27} \mathrm{H}_{18} \mathrm{~N}_{2} \mathrm{O}_{5}$ & 450.44 & Solid & Brown & 65.2 & $115-118$ & 0.60 & 5.82 \\
\hline
\end{tabular}

aSolvent system: n-Hexane/EtOAc - 3:2; bOctanol/water partition coefficient, calculated lipophilicity, determined using ChemDraw Ultra 10.0 software.

in $\mathrm{CH}_{3} \mathrm{CN}(5 \mathrm{ml})$ at room temperature in presence of p-TsOH (50 mol \%) for 6-8 h. After completion of reaction (as indicated by TLC and FT-IR), the solid obtained was separated by filtration, washed with ice-cold water followed by ice-cold ethanol, and dried to obtain the crude compound. The crude solid was recrystallized from ethyl acetate to get the pure product.

The physicochemical details including melting point $\left(\mathrm{MP},{ }^{\circ} \mathrm{C}\right)$ and TLC retention factor $(\mathrm{R} f)$ of synthesized compounds are presented in Table 1. The spectral data (UV, FT-IR, ${ }^{1} \mathrm{HNMR},{ }^{13} \mathrm{CNMR}$ and Mass) depicted below are in close agreement with structures of the synthesized compounds, $4 \mathbf{a}-\mathbf{j}$.

Compound 4a [2-Hydroxy-3-((4-methoxyphenyl) (propylamino)methyl)-1,4-naphthoquinone]: UV spectrum $\left(\mathrm{CH}_{3} \mathrm{CN}\right), \lambda_{\max }(\mathrm{nm}): 242.5$; IR (ATR), $v\left(\mathrm{~cm}^{-1}\right): 3512$ (O-H), $3334(\mathrm{~N}-\mathrm{H}), 3016,3012$ (C-H aryl), 2964, 2945, 2882, $2863\left(\mathrm{C}-\mathrm{H}, \mathrm{CH}_{3}\right.$ and $\left.\mathrm{CH}_{2}\right), 1667,1645$ (2 C=O), 1358 (C-N), 1223, 1121 (C-O); ${ }^{1} \mathrm{H}$ NMR (400 MHz, $\left.\mathrm{CDCl}_{3}\right), \delta$ (ppm): 9.64 (bs, $\left.1 \mathrm{H}, \mathrm{OH}\right), 7.94-7.85(\mathrm{~m}, 4 \mathrm{H}$, Ar-H), 6.42-6.38 (m, 4H, Ar-H), 4.50 (s, 1H, NH-alkyl), 3.11 (s, $3 \mathrm{H}, \mathrm{OCH}_{3}$ ), 3.42 (s, $\left.1 \mathrm{H}, \mathrm{C} \underline{\mathrm{H}}-\mathrm{NH}\right), 3.13$ (s, $2 \mathrm{H}$, $\left.\mathrm{NH}-\mathrm{CH}_{2}\right), 2.26\left(\mathrm{t}, 2 \mathrm{H}, \mathrm{J}=7.82 \mathrm{~Hz}, \mathrm{NH}-\mathrm{CH}_{2} \mathrm{CH}_{2}\right), 1.46$ (m, $\left.2 \mathrm{H}, \mathrm{CH}_{2} \mathrm{CH}_{2} \mathrm{CH}_{3}\right), 1.18\left(\mathrm{t}, 3 \mathrm{H}, \mathrm{J}=7.44 \mathrm{~Hz}, \mathrm{CH}_{2} \mathrm{CH}_{3}\right)$; ${ }^{13} \mathrm{C}$ NMR $\left(100 \mathrm{MHz}^{2} \mathrm{CDCl}_{3}\right), \delta$ (ppm): 182.24, 176.21 (2 $\mathrm{C}=\mathrm{O}), 164.28(\mathrm{C}-\mathrm{OH}), 140.24,138.22,132.01$, 128.56, 126.24, 122.04 (aromatic C), 96.67, 88.32 (olefinic $\mathrm{C}=\mathrm{C}$, naphthoquinone), $68.42(>\mathrm{CH}-\mathrm{NH})$, 62.12, 56.34, 44.20, 18.42 (aliphatic $\mathrm{CH}_{2}$ and $\mathrm{CH}_{3}$ ); MS (ESI), $\mathrm{m} / \mathrm{z}(\%): 352.01(100),[\mathrm{M}+\mathrm{H}]^{+}$.

Compound 4b [2-Hydroxy-3-((4-fluorophenylamino) (phenyl)methyl)-1,4-naphthoquinone]: UV spectrum $\left(\mathrm{CH}_{3} \mathrm{CN}\right), \lambda_{\max }(\mathrm{nm}): 245.5$; IR (ATR), $v\left(\mathrm{~cm}^{-1}\right): 3578$, $(\mathrm{O}-\mathrm{H}), 3315(\mathrm{~N}-\mathrm{H}), 3012,3010$ (C-H aryl), 2956, 2912, 2898, $2863\left(\mathrm{C}-\mathrm{H}, \mathrm{CH}_{3}\right.$ and $\left.\mathrm{CH}_{2}\right), 1636,1642(2 \mathrm{C}=\mathrm{O})$,
1260 (C-N), 1342 (C-F); ${ }^{1} \mathrm{H}$ NMR (400 MHz, $\mathrm{CDCl}_{3}$ ), $\delta$ (ppm): 9.86 (bs, 1H, OH), 8.00-7.82 (m, 4H, Ar-H),

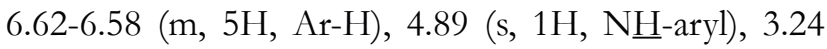
(s, $2 \mathrm{H}, \mathrm{CH}-\mathrm{NH}) ;{ }^{13} \mathrm{C}$ NMR $\left(100 \mathrm{MHz}, \mathrm{CDCl}_{3}\right), \delta$ (ppm): 186.23, 178.40 (2 C=O), $168.34(\mathrm{C}-\mathrm{OH}), 142.22,136.20$, 133.02, 128.24, 126.12, 122.13, 118.14, 116.12 (aromatic C), $96.78,88.45$ (olefinic $\mathrm{C}=\mathrm{C}$, naphthoquinone), 66.20 (>CH-NH); MS (ESI), m/z (\%): 374.12 (100), $[\mathrm{M}+\mathrm{H}]^{+}$. Compound 4c [2-Hydroxy-3-((4-nitrophenyl)(4-nitrophenylamino)methyl)-1,4-naphthoquinone]:UV spectrum $\left(\mathrm{CH}_{3} \mathrm{CN}\right), \lambda_{\max }(\mathrm{nm}): 275.5$; IR (ATR), $v\left(\mathrm{~cm}^{-1}\right): 3426$, $(\mathrm{O}-\mathrm{H}), 3338(\mathrm{~N}-\mathrm{H})$, 3010, 3008 (C-H aryl), 2902, 2896 (C-H, >CH-), 1668, 1659 (2 C=O), 1482, $1368\left(\mathrm{C}-\mathrm{NO}_{2}\right.$, aryl), $1282(\mathrm{C}-\mathrm{N}, \mathrm{C}-\mathrm{NH}) ;{ }^{1} \mathrm{H}$ NMR $\left(400 \mathrm{MHz}, \mathrm{CDCl}_{3}\right)$, $\delta$ (ppm): 9.16 (bs, 1H, OH), 8.21-7.98 (m, 4H, Ar-H), 6.42-6.34 (m, 4H, Ar-H), 6.28-6.24 (m, 4H, Ar-H), 4.76 (s, 1H, NH-aryl), 3.22 (s, 2H, Cㅍ-NH); ${ }^{13} \mathrm{C}$ NMR $(100$ $\left.\mathrm{MHz}, \mathrm{CDCl}_{3}\right), \delta$ (ppm): 184.12, $178.60(2 \mathrm{C}=\mathrm{O}), 168.20$, $162.12,156.12,148.20,140.20,138.42,132.12,130.40$ (aromatic C), 96.12, 88.19 (olefinic $\mathrm{C}=\mathrm{C}$, naphthoquinone), 65.27 (>CH-NH); MS (ESI), m/z (\%): 346.06 (100), $[\mathrm{M}+\mathrm{H}]^{+}$.

Compound 4d [2-Hydroxy-3-((2-hydroxyphenyl) (3-nitrophenylamino)methyl)-1,4-naphthoquinone]: UV spectrum $\left(\mathrm{CH}_{3} \mathrm{CN}\right), \lambda_{\text {max }}(\mathrm{nm}): 270.0 ; \mathrm{IR}(\mathrm{ATR}), v\left(\mathrm{~cm}^{-1}\right)$ : 3514, 3482, (2 O-H), 3332 (N-H), 3012, 3006 (C-H aryl), 2900, 2898 (C-H, >CH-), 1668, 1642 (2 C=O), 1434, $1382\left(\mathrm{C}-\mathrm{NO}_{2}\right.$, aryl), $1278(\mathrm{C}-\mathrm{N}, \mathrm{C}-\mathrm{NH}) ;{ }^{1} \mathrm{H}$ NMR (400 $\mathrm{MHz}, \mathrm{CDCl}_{3}$ ), $\delta$ (ppm): 9.16 (bs, $\left.1 \mathrm{H}, \mathrm{OH}\right), 9.02$ (bs, $1 \mathrm{H}$, aryl $\mathrm{OH}), 8.20-7.98(\mathrm{~m}, 4 \mathrm{H}, \mathrm{Ar}-\mathrm{H}), 6.49-6.44(\mathrm{~m}, 4 \mathrm{H}$, Ar-H), 6.38-6.32 (m, 4H, Ar-H), 4.78 (s, 1H, NH-aryl), 3.24 (s, $2 \mathrm{H}, \mathrm{CH}-\mathrm{NH}) ;{ }^{13} \mathrm{C}$ NMR $\left(100 \mathrm{MHz}, \mathrm{CDCl}_{3}\right), \delta$ (ppm): 182.00, 178.24 (2 C=O), 168.42, 164.20, 158.32, 152.42, 148.14, 144.12, 140.26, 138.23, 132.43, 128.20 (aromatic C), 96.02, 89.33 (olefinic $\mathrm{C}=\mathrm{C}$, naphthoqui- 
none), 65.45 (>CH-NH); MS (ESI), m/z (\%): 417.13 (100), $[\mathrm{M}+\mathrm{H}]^{+}$.

Compound 4e [2-Hydroxy-3-((3-nitrophenylamino) (phenyl)methyl))-1,4-naphthoquinone]: UV spectrum $\left(\mathrm{CH}_{3} \mathrm{CN}\right), \lambda_{\max }(\mathrm{nm}): 255.5$; IR (ATR), $v\left(\mathrm{~cm}^{-1}\right): 3502$ (O-H), 3348 (N-H), 3014, 3010 (C-H aryl), 2912, 2898 (C-H, >CH-), 1667, 1654 (2 C=O), 1512, $1445\left(\mathrm{C}-\mathrm{NO}_{2}\right.$, aryl), 1279 (C-N, C-NH); ${ }^{1} \mathrm{H}$ NMR $\left(400 \mathrm{MHz}, \mathrm{CDCl}_{3}\right)$, $\delta$ (ppm): 9.22 (bs, 1H, OH), 8.16-7.99 (m, 4H, Ar-H), 6.52-6.48 (m, 4H, Ar-H), 6.34-6.30 (m, 5H, Ar-H), 4.64 (s, 1H, NH-aryl), 3.20 (s, 2H, Cㅍ-NH); ${ }^{13} \mathrm{C}$ NMR (100 $\left.\mathrm{MHz}, \mathrm{CDCl}_{3}\right), \delta$ (ppm): 182.34, 178.26 (2 C=O), 168.29, 164.34, 158.20, 150.24, 148.29, 128.02, 126.24 (aromatic C), 96.46, 89.45 (olefinic $\mathrm{C}=\mathrm{C}$, naphthoquinone), 66.02 (>CH-NH); MS (ESI), m/z (\%): $402.01(100),[\mathrm{M}+\mathrm{H}]^{+}$. Compound 4f [2-Hydroxy-3-((4-hydroxyphenyl) (3-nitrophenylamino)methyl)-1,4-naphthoquinone]: UV spectrum $\left(\mathrm{CH}_{3} \mathrm{CN}\right), \lambda_{\text {max }}(\mathrm{nm}): 260.5 ; \mathrm{IR}$ (ATR), $v\left(\mathrm{~cm}^{-1}\right)$ : 3534, 3480, (2 O-H), 3345 (N-H), 3012, 3010 (C-H aryl), 2902, 2899 (C-H, >CH-), 1668, 1657 (2 C=O), 1438, $1385\left(\mathrm{C}-\mathrm{NO}_{2}\right.$, aryl), 1277 (C-N, C-NH); ${ }^{1} \mathrm{H}$ NMR (400 $\mathrm{MHz}, \mathrm{CDCl}_{3}$ ), $\delta$ (ppm): 9.12 (bs, $\left.1 \mathrm{H}, \mathrm{OH}\right), 9.06$ (bs, $1 \mathrm{H}$, aryl $\mathrm{OH}), 8.22-8.12(\mathrm{~m}, 4 \mathrm{H}, \mathrm{Ar}-\mathrm{H}), 6.42-6.40(\mathrm{~m}, 4 \mathrm{H}$, Ar-H), 6.36-6.34 (m, 4H, Ar-H), 4.62 (s, 1H, NH-aryl), 3.42 (s, 2H, CH-NH); ${ }^{13} \mathrm{C}$ NMR (100 MHz, $\left.\mathrm{CDCl}_{3}\right), \delta$ (ppm): 182.14, 178.54 (2 C=O), 168.20, 166.24, 158.26, 152.24, 148.26, 144.20, 142.26, 138.43, 130.12, 128.40 (aromatic C), 98.24, 88.10 (olefinic $\mathrm{C}=\mathrm{C}$, naphthoquinone), 68.42 (>CH-NH); MS (ESI), m/z (\%): 417.00 (100), $[\mathrm{M}+\mathrm{H}]^{+}$.

Compound 4g [2-Hydroxy-3-(phenyl(pyrrolidin1-ylamino)methyl)-1,4-naphthoquinone]: UV spectrum $\left(\mathrm{CH}_{3} \mathrm{CN}\right), \lambda_{\max }(\mathrm{nm}): 240.5$; IR (ATR), $v\left(\mathrm{~cm}^{-1}\right): 3520$ $(\mathrm{O}-\mathrm{H}), 3342(\mathrm{~N}-\mathrm{H}), 3008,3004$ (C-H aryl), 2920, 2899 (C-H, >CH-), 1658, 1644 (2 C=O), $1262(\mathrm{C}-\mathrm{N}$, $\mathrm{C}-\mathrm{NH}) ;{ }^{1} \mathrm{H}$ NMR $\left(400 \mathrm{MHz}, \mathrm{CDCl}_{3}\right), \delta$ (ppm): 9.20 (bs, 1H, OH), 8.21-7.94 (m, 4H, Ar-H), 6.18-6.14 (m, 5H, Ar-H), 4.02 (s, 1H, NH-(pyrrolidin-1-yl)), 3.22 (s, 2H, CH-NH), 2.62-2.44 (m, 8H, $\left(\mathrm{CH}_{2}\right)$, pyrrolidin-1-yl); ${ }^{13} \mathrm{C}$ NMR (100 MHz, $\left.\mathrm{CDCl}_{3}\right), \delta$ (ppm): 184.24, 178.06 (2 C=O), 138.23, 132.43, 128.20, 126.20, 122.42 (aromatic $\mathrm{C}$ ), 96.24, 89.26 (olefinic $\mathrm{C}=\mathrm{C}$, naphthoquinone), 65.64 (>CH-NH), 48.24, 42.12, 28.04, $26.12\left(\mathrm{CH}_{2}\right.$, pyrrolidin-1-yl); MS (ESI), m/z (\%): 349.14 (100), [M+H] $]^{+}$ Compound 4h [2-Hydroxy-3-((4-nitrophenyl)(pyrrolidin1-ylamino)methyl)-1,4-naphthoquinone]: UV spectrum $\left(\mathrm{CH}_{3} \mathrm{CN}\right), \lambda_{\max }(\mathrm{nm}): 255.5$; IR (ATR), $v\left(\mathrm{~cm}^{-1}\right): 3520$ $(\mathrm{O}-\mathrm{H}), 3342(\mathrm{~N}-\mathrm{H}), 3008,3004$ (C-H aryl), 2920, 2899 (C-H, >CH-), 1658, 1644 (2 C=O), 1542, 1448 (C-NO, aryl), $1262(\mathrm{C}-\mathrm{N}, \mathrm{C}-\mathrm{NH}) ;{ }^{1} \mathrm{H} \mathrm{NMR}\left(400 \mathrm{MHz}, \mathrm{CDCl}_{3}\right)$, $\delta$ (ppm): 9.20 (bs, 1H, OH), 8.21-7.94 (m, 4H, Ar-H), 6.38-6.32 (m, 4H, Ar-H), 4.24 (s, 1H, NH-(pyrrolidin- 1-yl)), 3.36 (s, 2H, C$-\mathrm{HH}), 2.64-2.42\left(\mathrm{~m}, 8 \mathrm{H},\left(\mathrm{CH}_{2}\right)_{4}\right.$, pyrrolidin-1-yl); ${ }^{13} \mathrm{C}$ NMR (100 MHz, $\left.\mathrm{CDCl}_{3}\right), \delta$ (ppm): 184.26, 178.00 (2 C=O), 158.24, 154.20, 150.34, 148.06, 142.22, 132.45, 128.24 (aromatic C), 96.02, 89.16 (olefinic $\mathrm{C}=\mathrm{C}$, naphthoquinone), $66.24(>\mathrm{CH}-\mathrm{NH})$, 48.20, 44.20, 28.24, $26.15\left(\mathrm{CH}_{2}\right.$, pyrrolidin-1-yl); MS (ESI), m/z (\%): 393.97 (100), [M+H] $]^{+}$.

Compound 4i [2-Hydroxy-3-((2-hydroxyphenyl) (pyrrolidin-1-ylamino)methyl)-1,4-naphthoquinone]: UV spectrum $\left(\mathrm{CH}_{3} \mathrm{CN}\right), \lambda_{\max }(\mathrm{nm}): 250.0$; IR (ATR), $v$ $\left(\mathrm{cm}^{-1}\right)$ : 3520, $3426(2 \mathrm{O}-\mathrm{H}), 3336(\mathrm{~N}-\mathrm{H}), 3012,3010$ (C-H aryl), 2912, 2888 (C-H, >CH-), 1676, 1642 (2 C=O), $1266(\mathrm{C}-\mathrm{N}, \mathrm{C}-\mathrm{NH}) ;{ }^{1} \mathrm{H}$ NMR (400 MHz, $\left.\mathrm{CDCl}_{3}\right), \delta$ (ppm): 9.22 (bs, 1H, OH), 9.12 (bs, 1H, aryl $\mathrm{OH})$, 8.02-7.96 (m, 4H, Ar- $\mathrm{H}), 6.36-6.34(\mathrm{~m}, 4 \mathrm{H}$, Ar-H), 4.26 (s, 1H, NH-(pyrrolidin-1-yl)), 3.34 (s, 2H, $\mathrm{CH}-\mathrm{NH}), 2.56-2.52$ (m, 8H, $\left(\mathrm{CH}_{2}\right)_{4}$, pyrrolidin-1-yl); ${ }^{13} \mathrm{C}$ NMR (100 MHz, $\left.\mathrm{CDCl}_{3}\right), \delta$ (ppm): 188.20, 182.12 (2 C=O), 158.20, 156.12, 152.68, 148.16, 144.80, 136.00, 128.01 (aromatic C), 96.49, 88.02 (olefinic $\mathrm{C}=\mathrm{C}$, naphthoquinone), 68.12 (>CH-NH), 48.05, 44.16, 28.02, $26.47\left(\mathrm{CH}_{2}\right.$, pyrrolidin-1-yl); MS (ESI), m/z (\%): 365.11 (100), $[\mathrm{M}+\mathrm{H}]^{+}$.

Compound 4j [2-hydroxy-3-(naphthalen-1-yl(3-nitrophenylamino)methyl)-1,4-naphthoquinone]:UV spectrum $\left(\mathrm{CH}_{3} \mathrm{CN}\right), \lambda_{\max }(\mathrm{nm}): 255.5$; IR (ATR), $v\left(\mathrm{~cm}^{-1}\right): 3524$ (O-H), $3338(\mathrm{~N}-\mathrm{H}), 3009,3006$ (C-H aryl), 2910, 2892 (C-H, >CH-), 1652, 1638 (2 C=O), 1544, 1428 (C-NO , aryl), 1264 (C-N, C-NH); ${ }^{1} \mathrm{H}$ NMR (400 $\left.\mathrm{MHz}_{2} \mathrm{CDCl}_{3}\right)$, $\delta$ (ppm): 9.24 (bs, 1H, OH), 8.22-7.98 (m, 4H, Ar-H), 6.36-6.34 (m, 4H, Ar-H), 4.22 (s, 1H, NH-aryl), 3.28 (s, $2 \mathrm{H}, \mathrm{CH}-\mathrm{NH}) ;{ }^{13} \mathrm{C}$ NMR $\left(100 \mathrm{MHz}, \mathrm{CDCl}_{3}\right), \delta$ (ppm): 182.42, 178.16 (2 C=O), 162.04, 158.14, 156.24, 140.12, 148.04, 140.23, 130.35, 128.08 (aromatic C), 94.12, 86.15 (olefinic $\mathrm{C}=\mathrm{C}$, naphthoquinone), $64.24(>\mathrm{CH}-\mathrm{NH})$; MS (ESI), m/z (\%): 451.12 (100), [M+H] $]^{+}$

\section{Antimalarial activity evaluation}

The in vitro antimalarial activity screening was carried out against laboratory adapted strains of $P$. falciparum by Giemsa stained slide method. ${ }^{21,22,23}$ Two strains were used for the study, one is CQ-sensitive 3D-7 and the other is CQ-resistant RKL-2. All the test compounds, $4 a-j$ were initially screened at two fixed doses, $5 \mu \mathrm{g} / \mathrm{ml}$ and $50 \mu \mathrm{g} / \mathrm{ml}$ against CQ-sensitive strain. Five compounds which showed considerably good activity in screening doses were further subjected to testing at multiple doses against both CQ-sensitive and CQ-resistant strains of P. falciparum. The in vitro screening study was carried out at the Department of Pharmaceutical Sciences, Dibrugarh University, Dibrugarh, Assam (India). 
Continuous culture of $P$. falciparum strain was maintained in vitro in $\mathrm{O}^{+}$human red blood cells diluted to 6\% HCT in RPMI-1640 medium supplemented with $25 \mathrm{mM}$ HEPES, $1 \%$ D-glucose, $0.23 \%$ sodium bicarbonate, gentamicin $(40 \mu \mathrm{g} / \mathrm{ml})$, amphotericin-B $(0.25 \mu \mathrm{g} / \mathrm{ml})$, and $10 \%$ human $\mathrm{AB}^{+}$serum. Incubations were done at $37^{\circ} \mathrm{C}$ and $5 \% \mathrm{CO}_{2}$ level in a modular incubator. D-sorbitol synchronized 1\% ring stage parasitaemia in 3\% HCT was used for antimalarial assays using 96 well microtitre plate. A stock solution of $5 \mathrm{mg} / \mathrm{ml}$ of the test compound was prepared in DMSO and subsequent dilutions were made with incomplete RPMI in duplicate. Each test well of the microtitre plate contained $20 \mu \mathrm{l}$ of the compound and $180 \mu \mathrm{l}$ of $1 \%$ ring stage parasitaemia in 3\% HCT. In addition, drug free negative control to assess the parasite growth and chloroquine diphosphate as positive control to assess the integrity of the assay were also maintained in duplicate in the microtitre plate. After $40 \mathrm{~h}$ of incubation at $37^{\circ} \mathrm{C}$ the smears were prepared from each well, stained with 3\% Giemsa and scanned under light microscope to ascertain percentage dead rings and schizonts by examining a minimum of 200 asexual parasites.

Test results were compared with the standard result of CQ. Each test compound was assayed in two replicates and counted (number of dead rings and schizonts) against 200 asexual parasites per replicate. The mean of percentage inhibition (\%dead rings + schizonts) of parasite growth was calculated for duplicate studies. Further, the $\mathrm{IC}_{50}$ values (in $\mu \mathrm{g} / \mathrm{ml}$ ) were also calculated from mean number of dead rings and schizonts for each tested compound using the NonLin $v 1.1$ software. ${ }^{24,25}$ The $\mathrm{IC}_{50}$ value represents the concentration of compound that inhibits the growth of malaria parasite by $50 \%$.

\section{RESULTS AND DISCUSSION}

\section{Chemistry}

A new series of Mannich base substituted lawsone derivatives were synthesized and evaluated for in vitro antimalarial activity. Several aliphatic/aromatic/heteroaromatic primary/secondary amines and different aromatic aldehydes were used for different Mannich substitutions on the structural scaffold 2-hydroxy-1,4-naphthoquinone (Lawsone). The target Mannich bases of lawsone are obtained as 3-alkyl/aryl/heteroaryl substituted 2-hydroxyaminonaphthquinones. Mannich reaction of lawsone, 1 with various aldehydes, $\mathbf{2}$ and amines, $\mathbf{3}$ was carried out in acetonitrile $\left(\mathrm{CH}_{3} \mathrm{CN}\right)$ at room temperature using $p$-toluene sulfonic acid ( $p$-Ts $\mathrm{OH}$ ) as catalyst, which yielded Mannich bases of lawsone as 3-alkyl/aryl/ heteroaryl substituted aminonaphthoquinones, 4a-j

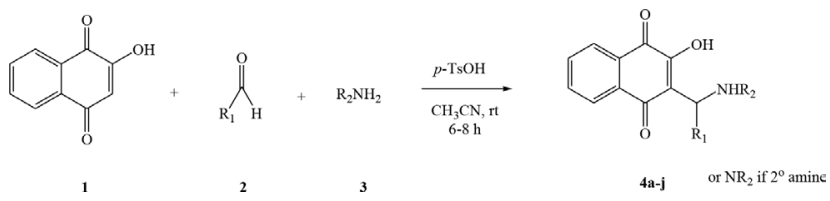

Scheme 1: Synthesis of lawsone Mannich bases; 4a: $R_{1}=$ p-methoxyphenyl, $R_{2}=n$-propyl; $4 b: R_{1}=$ phenyl, $R_{2}=p$-fluorophenyl; 4c: $R_{1}=p$-nitrophenyl, $R_{2}=m$-nitrophenyl; 4d: $\mathbf{R}_{1}=$ o-hydroxyphenyl, $\mathbf{R}_{2}=\boldsymbol{m}$-nitrophenyl; $4 \mathrm{e}: \mathbf{R}_{1}=$ phenyl, $R_{2}=m$-nitrophenyl; 4f: $R_{1}=p$-hydroxyphenyl, $R_{2}=m$-nitrophenyl; 4g: $R_{1}=$ phenyl, $R_{2}=1$-pyrrolidinyl; $4 \mathrm{~h}: \mathbf{R}_{1}=p$-nitrophenyl, $\mathbf{R}_{2}=$ 1-pyrrolidinyl; 4i: $\mathbf{R}_{1}=$ o-hydroxyphenyl, $\mathbf{R}_{2}=$ 1-pyrrolidinyl; 4j: $R_{1}=1$-naphthyl, $R_{2}=m$-nitrophenyl.

(Scheme 1). Prior to reaction, optimization studies were performed in terms different reaction conditions which included time of reaction, and solvent and reagent/ catalyst used. The reaction conditions as described in experimental section afforded best results with higher yield for a shorter reaction time. All the compounds (4a-j) were obtained in good yields with high purity.

The spectral data (UV, FT-IR, ${ }^{1} \mathrm{HNMR},{ }^{13} \mathrm{CNMR}$ and Mass) depicted in experimental section are in close agreement with structures of the synthesized compounds, 4a-j. UV spectra (in acetonitrile) exhibited characteristic absorption maxima with $\lambda_{\text {max }}$ ranging from 240.5 - 275.5 $\mathrm{nm}$, which indicates the presence of chromophoric 2-hydroxy-1,4-naphthoquinone system in the structure of compounds, $4 \mathbf{a}-\mathbf{j}$. The shift of absorption maxima towards longer wave length is observed due to presence of strong auxochromes like nitro and hydroxy groups in the structure of compounds like $4 \mathrm{c}\left(\lambda_{\max }=275.5 \mathrm{~nm}\right)$, $4 \mathbf{d}\left(\lambda_{\max }=270.0 \mathrm{~nm}\right)$ and $4 \mathrm{f}\left(\lambda_{\max }=260.0 \mathrm{~nm}\right)$. The infrared spectral data showed characteristic absorption bands for hydroxyl (O-H str., broad and strong), $2^{\circ}$ amino (N-H str., weak), carbonyl $(\mathrm{C}=\mathrm{O}$ str., sharp and strong) and aryl nitro (N-O str., strong) groups at their corresponding frequencies $\left(\mathrm{v}, \mathrm{cm}^{-1}\right)$ as described above. Additionally, peaks due to C-H (alkyl/aryl), C-N (alkyl/aryl), C-O (aryl) and C-F (aryl) stretching frequencies already described above were also observed in the IR spectra of synthesized compounds, which ascertain the anticipated structure of $4 \mathbf{a}-\mathbf{j} .{ }^{1} \mathrm{H}$ NMR spectra displayed signals ( $\delta$ values, ppm) for different structural protons of naphthoquinone ring system as well as of the Mannich base component such as substituted $\left(\mathrm{F}, \mathrm{NO}_{2}, \mathrm{OH}\right)$ phenyl or 1-pyrrolidinyl ring systems of synthesized compounds. The appearance of characteristic proton chemical shifts depicted in experimental section for $\mathrm{OH}, \mathrm{NH}, \mathrm{CH}, \mathrm{OCH}_{3}$ and $\mathrm{CH}_{2}$ groupings confirm their presence in the structure of respective compounds. ${ }^{15} \mathrm{~A}$ broad singlet of one proton due to hydroxyl group was appeared in the range of 9.12-9.86 ppm for all the synthesized compounds, $4 \mathbf{a}-\mathbf{j}$. Depending on 


\begin{tabular}{|c|c|c|c|c|c|c|c|c|c|c|}
\hline \multirow[t]{2}{*}{ Dose $(\mu \mathrm{g} / \mathrm{ml})$} & \multicolumn{10}{|c|}{ (\%Dead rings + Schizonts) ${ }^{\mathrm{b}}$} \\
\hline & $4 a$ & $4 b$ & $4 c$ & $4 d$ & $4 e$ & $4 f$ & $\mathbf{4 g}$ & $4 h$ & $4 i$ & $4 j$ \\
\hline 5 & 10.5 & 21.0 & 35.0 & 31.2 & 8.00 & 12.2 & 29.0 & 15.5 & 32.0 & 15.0 \\
\hline 50 & 38.2 & 65.0 & 72.5 & 75.0 & 25.5 & 30.0 & 69.5 & 42.5 & 70.0 & 34.0 \\
\hline
\end{tabular}

aagainst CQ-sensitive P. falciparum strain; bmean of two replicate observations (counted against 200 cells per replicate)

\begin{tabular}{|c|c|c|c|c|c|c|}
\hline \multicolumn{7}{|c|}{ Table 3: Antimalarial activity data at multiple doses. } \\
\hline \multirow{2}{*}{ Strain } & \multicolumn{7}{|c|}{ IC $_{\mathbf{5 0}}(\boldsymbol{\mu g} / \mathbf{m l})^{\mathbf{a}}$} \\
\cline { 2 - 8 } & $\mathbf{4 b}$ & $\mathbf{4 c}$ & $\mathbf{4 d}$ & $\mathbf{4 g}$ & $\mathbf{4 i}$ & $\mathbf{C Q}^{\mathbf{b}}$ \\
\hline 3D-7 (CQ-Sensitive) & 0.423 & 0.475 & 0.502 & 0.412 & 0.411 & 0.044 \\
\hline RKL-2 (CQ-Resistant) & 1.492 & 1.391 & 2.394 & 2.212 & 1.170 & 0.216 \\
\hline
\end{tabular}

acalculated using NonLin v1.1 software and mean of two replicate observations (counted against 200 cells per replicate), bstandard drug

the nature ubstitution patterns at $\mathrm{CH}\left(\mathrm{R}_{1}\right)$, the aromatic protons of compounds $\mathbf{4 a - j}$ were observed in distinct signals with expected multiplet patterns integrating more than one proton due to the close chemical shifts ranging from 6.18 to $6.52 \mathrm{ppm}$. Aromatic protons of naphthoquinone moiety were also appeared as multiplets with chemical shifts at range of 7.98-8.22 for the synthesized compounds. Singlet at 4.02, 4.26 and $4.64 \mathrm{ppm}$ were assigned to one proton of $\mathrm{NH}$-alkyl, $\mathrm{NH}$-pyrrolidin-1-yl and NH-aryl, respectively, for certain compounds. Singlets in the range of 3.22-3.42 were also observed for one methane proton of $\mathrm{CH}(\mathrm{NH})$ group for all compounds. Moreover, characteristic singlet, triplet or multiplet peaks were appeared in the aliphatic regions for methoxyl, methyl and/or methylene groups(s) of alkyl chain and methylene groups of pyrrolidin-1-yl ring system as depicted in the experimental section. The ${ }^{13} \mathrm{C}$ resonance data revealed the presence of different carbon atoms such as aryl- $\mathrm{C}, \mathrm{C}=\mathrm{O}$, olefinic $>\mathrm{C}=\mathrm{C}<$ of naphthoquinone system, $>\mathrm{CHNH}-, \mathrm{CH}_{2}$ and $\mathrm{CH}_{3}$ in the basic structural scaffold of molecules including 1,4-napththoquinoe nucleus, substituted aryl moieties/unsubstituted heteroaryl (1-pyrrolidinyl) and the aminomethyl bridge ( $>\mathrm{CH}$ (aryl)-NH-aryl/heteroaryl).$^{15}$ The characteristic signals in the region of $188.20-178.00 \mathrm{ppm}$ and 168.34-164.28 ppm were assigned for carbons of $\mathrm{C}=\mathrm{O}$ and $\mathrm{C}(\mathrm{OH})$ groups, respectively. Aromatic carbons were appeared at range 168.42-116.12 ppm and aliphatic carbons $\left(\mathrm{CH}_{3}, \mathrm{CH}_{2}\right)$ were observed in the region of 62.12-18.42 ppm. Distinct signals due to $\mathrm{NH}$ proton of $>\mathrm{CH}-\mathrm{NH}$ moiety was observed with chemical shift ranging from 68.42-65.27. Olefinic $(\mathrm{CH}=\mathrm{CH})$ protons were appeared in the region of 98.24-86.15. Downfield signals for methylene protons of pyrrolidin-1-yl ring system were appeared at range of 48.20-26.15. The mass spectra of 4 a-f exhibited prominent molecular ion peaks, $[\mathrm{M}+\mathrm{H}]^{+}$which are in accordance with the anticipated mass corresponding to their respective molecular formula.

\section{Antimalarial activity}

All the synthesized compounds, 4a-j showed activity against CQ-sensitive P. falciparum strain in a dose dependant manner at the screening doses, $5 \mu \mathrm{g} / \mathrm{ml}$ and $50 \mu \mathrm{g} / \mathrm{ml}$ with some degree of variation. The mean antimalarial activity (\%dead rings + schizonts) data for two replicate observations at screening doses against CQ-sensitive P. falciparum strain is presented in Table 2 . Results clearly reveal that five compounds (4b, $4 c, 4 d, 4 g$ and $4 \mathbf{i})$ exhibited comparatively better activity than other synthesized analogues (4a, $\mathbf{4 e}, \mathbf{4 f}, \mathbf{4 h}$ and $\mathbf{4 j})$. For these compounds, the percentage inhibitory activities range from $8.0-35.0 \%$ and $65.0-75.0 \%$ at screening doses of $5 \mu \mathrm{g} / \mathrm{ml}$ and 50 $\mu \mathrm{g} / \mathrm{ml}$, respectively. Further testing at multiple doses these five compounds showed good activities against both sensitive and resistant (RKl-2) strains, which, however, was considerably less than that of the standard reference drug, CQ. The activity data $\left(\mathrm{IC}_{50}\right.$ in $\left.\mu \mathrm{g} / \mathrm{ml}\right)$ at multiple doses is depicted in Table 3. Table 3 reflects that the antimalarial activity of tested compounds was found better on sensitive strain $\left(\mathrm{IC}_{50}=0.411-0.502 \mu \mathrm{g} / \mathrm{ml}\right)$ than the resistant one $\left(\mathrm{IC}_{50}=1.391-2.394 \mu \mathrm{g} / \mathrm{ml}\right)$. The $\mathrm{IC}_{50}$ values for CQ were $0.044 \mu \mathrm{g} / \mathrm{ml}$ and $0.216 \mu \mathrm{g} / \mathrm{ml}$ against sensitive and resistant strains, respectively. However, compound $4 \mathrm{c}$ and compound $4 \mathbf{i}$ exhibited superior antimalarial potency over rest of the analogues, i.e., compound $\mathbf{4 b}$, compound $\mathbf{4 d}$ and compound $\mathbf{4 g}$.

From above results it is clear that newly synthesized lawsone Mannich base derivatives possess antimalarial potential. Different structural substitutions such as non-bulky alkyl (propyl), bulky aryl (phenyl, 1-naphthyl, $p$-fluorophenyl, $m$-nitrophenyl, o-hydroxyphenyl and p-hydroxyphenyl), and bulky heteroaryl (1-pyrrolidinyl) 
groups at C-3 position of the 1,4-naphthoquinone ring system have contributing effects towards either increasing or decreasing the antimalarial efficacy of the lawsone scaffold, 2-hydroxy-1,4-naphthoquinone system. Some degree of variations in activity among synthesized analogues at screening doses might be due to different Mannich substitution pattern. From literature, ${ }^{5,16}$ it is apparent that the presence of an alkyl substituted amino methyl group with respect to ortho hydroxyl function in the phenyl ring of quinoline side chain moiety is believed to be important for antiparasitic or resistant preventing property of amodiaquine or isoquine. Our present studies is also consistent with earlier investigation claiming that molecules with naphthoquinone structure with the incorporation of an amino groups often results in increased biological activities including antimalarial activity. ${ }^{14}$

Moreover, structure-activity relationship (SAR) studies indicate that bulky aryl (phenyl) moiety with electronwithdrawing $\left(\mathrm{NO}_{2}, \mathrm{~F}\right)$ or electron-releasing $(\mathrm{OH}$, $\mathrm{OCH}_{3}$ ) function is likely more important for the activity in comparison to heteroaryl like 1-pyrrolidinyl and small alkyl $\left(\mathrm{C}_{3} \mathrm{H}_{7}\right)$ groups. The aminomethyl $(>\mathrm{CH}-\mathrm{NH}-)$ bridge between two bulky aromatic groups is also considered to be an important requirement for the activity. Substitutions at ortho or para have greater effects than meta substitution. Electron donating group like $\mathrm{OH}$ have much stronger effects than electron withdrawing group like $\mathrm{NO}_{2}$. It is observed that di-substitution is less important for activity than mono-substitution. Because of this reason, compound $\mathbf{4 i}$ is more potent than compound $4 \mathbf{c}$. The antimalarial activity of newly reported aminonaphthoquinones could be attributed due to 1,4-naphthoquinone system (involving two carbonyl sites) mediated redox reactions/alkylation mechanism in the parasite. ${ }^{11,12}$

Lipophilicity is an important criterion for a molecule to exhibit desired biological activity. A molecule with optimum lipophilicity readily penetrates biological membrane that lead to attain an intracellular concentration favourable for desired activity. Therefore, a good drug molecule possesses balanced relationship between lipophilicity and drug activity. ${ }^{17,18,19,20}$ In our study, the lipophilicity expressed as $\operatorname{cog} \mathrm{P}_{\mathrm{o} / \mathrm{w}}$ (calculated oil/water partition coefficient) value presented in Table 1 indicated that higher the value of $\operatorname{cog} \mathrm{P}$, better the antimalarial activity was observed. The compound $4 \mathbf{i}$ having optimal lipophilicity with $\operatorname{clog} \mathrm{P}$ value of 2.88 showed best antimalarial activity in the series. Rest of the compounds (4a-h, $4 \mathbf{i})$ possess either less sufficient or more than optimal lipophilicity $(\operatorname{cog} \mathrm{P}=2.88)$ which perhaps the reason behind their reduced activity than the compound 4i. Compound $4 \mathbf{i}$ with 4-hydroxyphenyl and 1-pyrrolidinyl bulky substituents possessed optimal lipophilicity which exhibited better permeability across parasitic cell membrane than rest of the analogues having other aryl or heteroaryl bulky substituents with either more or less than optimal lipophilicity.

\section{CONCLUSION}

Newly reported lawsone Mannich bases can be regarded as either semi-synthetic derivatives of lawsone or synthetic aminonaphthoquinones. They are derived from bioactive scaffold of 2-hydroxy-1,4-naphthoquinone (residue of atovaquone) after minor structural modification. Lawsone Mannich bases with bulky aryl/heteroaryl substitution exhibited good antimalarial activity. SAR study suggests that antimalarial potential of lawsone structural scaffold can be modulated by different substitution pattern like Mannich base substitution (alkyl/aryl/heteroaryl substituted aminoalkyl moiety) at the C-3 position of 1,4-naphthoquinone ring system. However, because of having antimalarial effectiveness, lawsone or lawsone derived aminonaphthoquinones can be used as lead structure(s) in the development of yet more potent antimalarial agents.

\section{ACKNOWLEDGEMENT}

Authors are thankful to the Director, SAIF, Panjab University, Chandigarh (India) for providing the spectral data of synthesized compounds.

\section{CONFLICT OF INTEREST}

Authors declare that there is no conflict of interest.

\section{ABBREVIATIONS}

TLC: Thin layer chromatography; UV: Ultraviolet spectroscopy; ACT: Attenuated Total Reflection; FT-IR: Fourier transform infrared spectroscopy; FT-NMR: Fourier transform nuclear magnetic resonance spectroscopy; TMS: Tetramethylsilane; $\mathbf{C D C l}_{3}$ : Deuterated chloroform; MS: Mass spectrometry; ESI-MS: Electrospray ionization-Mass Spectrometer; Q-TOF: Quadrupole-Time of flight; HCT: Haematocrit; p-TsOH: para-Toluene sulfonic acid; RPMI: Roswell Park Memorial Institute; HEPES: (N-2-hydroxyethylpiperazine-N2-ethane sulfonic acid).

\section{REFERENCES}

1. World Malaria Report. World Health Organization: Geneva. 2016.

2. Rudrapal M, Chetia D. Endoperoxide antimalarials: development,structural diversity and pharmacodynamic aspects with reference to 1,2,4-trioxanebased structural scaffold. Drug Des Dev Ther. 2016;10:3575-90.

3. Rudrapal M, Chetia D, Singh V. Novel series of 1,2,4-trioxane derivatives as antimalarial agents. J. Enzyme Inhib Med Chem. 2017;32(1):1159-73. 
4. Jinky G, Dipak C, Mukesh KK, Mithun R. Synthesis and antimalarial activity evaluation of some mannich bases of tetraoxane-phenol conjugate. Indian J Pharm Edu Res. 2016;50(4):591-7.

5. Sharma D, Chetia D, Rudrapal M. Design, synthesis and antimalarial activity of some new 2-hydroxy-1,4-naphthoquinone- 4-hydroxyaniline hybrid Mannich bases. Asian J Chem. 2016;28(4):782-8.

6. Rudrapal M, Chetia D. Plant flavonoids as potential source of future antimalarial leads. Sys Rev Pharm. 2017;8(1):13-18.

7. Dinio T, Gorka AP, McGinniss A, Roepe PD, Morgan JB. Investigating the activity of quinine analogues versus chloroquine resistant Plasmodium falciparum. Bioorg Med. Chem. 2012;20(10):3292-7.

8. Meshnick SR. Artemisinin: mechanisms of action, resistance and toxicity. Int J Parasitol. 2002;32(13):1655-60.

9. Singh KD, Laqmana S, Mathur KA. Lawsonia inermis L. - A commercially important primaeval dying and medicinal plant with diverse pharmacological activity: A review. Ind Crop Prod. 2015;65:269-86.

10. Pradhan R, Dandawate P, Vyas A, Padhye S, Biersack B, Schobert R, et al. From body art to anticancer activities: perspectives on medicinal properties of henna. Curr Drug Targets. 2012;13(14):1777-98.

11. Rezende LCDD, Fumagalli F, Bortolin MS, Oliveira MGD, Paula MHD, Andrade-Neto VFD, et al. In vivo antimalarial activity of novel 2-hydroxy3-anilino-1,4-naphthoquinones obtained by epoxide ring-opening reaction. Bioorg Med Chem. 2013;23(16):4583-6.

12. Pingaew R, Prachayasittikul V, Worachartcheewan A, Nantasenamat $C$, Prachayasittikul S, Ruchirawat $\mathrm{S}$, et al. Novel 1,4-naphthoquinone-based sulfonamides: Synthesis, QSAR, anticancer and antimalarial studies. Eur J Med Chem. 2015;103:446-59.

13. Lamoureux G, Perez AL, Araya M, Agüero C. Reactivity and structure derivatives of 2-hydroxy-1,4-naphthoquinone (lawsone). J Phys Org Chem. 2008;21(12):1022-8.

14. Rodolfo GF, João F, Allochio F, Thieres MCP, Valdemar LJ, Reginaldo BDS, et al. A simple and convenient method for synthesis of new

\section{PICTORIAL ABSTRACT}

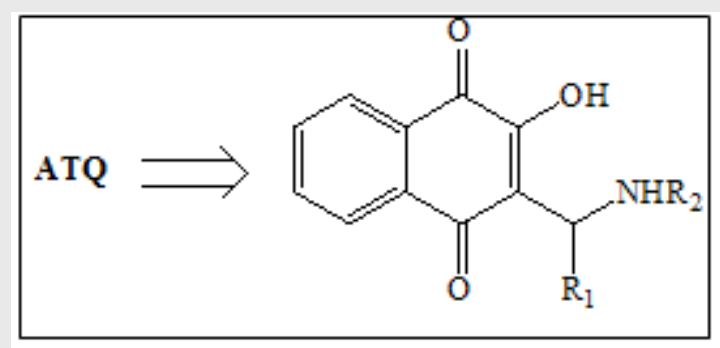

aminonaphthoquinones derived from lawsone by catalytic multicomponent Mannich reaction. Tetrahedron Lett. 2014;55(31):4373-7.

15. Silverstein RM, Webster FX, editors. Spectrometric identification of organic compounds. New York: John Wiley and Sons Ltd. 2014.

16. O'Neil PM, Mukhtar A, Stocks PA, Randle LE, Hindley S, Ward SA, et al. Isoquine and related amodiaquine analogues: a new generation of improved 4-aminoquinoline antimalarials. J Med Chem. 2003;46(23):4933-45.

17. Lipinski CA, Lombardo F, Dominy BW, Feeney PJ. Experimental and computational approaches to estimate solubility and permeability in drug discovery and development settings. Adv Drug Deliv Rev. 2001;46(1-3):3-26.

18. Rudrapal M, Chetia D. QSAR Analysis of 7-Chloro-4-Aminoquinoline Derivatives as Antimalarial Agents. Asian J Org Med Chem. 2016:1(2):51-54.

19. Rudrapal M, Chetia D. QSAR study of trioxane derivatives as antimalarial agents. Curr Trends Pharm Res. 2017;3(1):1-17.

20. Kerns EH, Di L, editors. Drug-like properties: concepts, structure design and methods from ADME to toxicity optimization. New York: Academic Press. 2015.

21. Sharma R, Goswami A, Rudrapal M, Sharma D, Sharma HK, Chetia D. In vitro evaluation of the antimalarial activity of a designed novel quinuclidine derivative. Curr Sci. 2016;111(12):2028-30.

22. Rudrapal M, Banu ZW, Chetia D. Newer series of trioxane derivatives as potent antimalarial agents. Med Chem Res. 2017;1-6. In Press. DOI 10.1007/ s00044-017-2090-8.

23. Roy S, Chetia D, Rudrapal M, Prakash M. Synthesis and antimalarial activity study of some new Mannich bases of 7-chloro-4-aminoquinoline. Med Chem. 2013;9(3):379-83.

24. Rudrapal M, Chetia D, Prakash M. Synthesis, antimalarial- and antibacterial activity evaluation of some new 4-aminoquinoline derivatives. Med Chem Res. 2013;22(8):3703-11.

25. Kashyap A, Chetia D, Rudrapal M. Synthesis, antimalarial activity evaluation and drug-likeness study of some new quinoline-lawsone hybrids. Indian J Pharm Sci. 2016;78(6):801-9.

\section{SUMMARY}

- A new series of lawsone Mannich base derivatives were synthesized, characterized and evaluated for in vitro antimalarial activity. Among synthesized compounds, five compounds showed good antimalarial activity against both chloroquine (CQ)sensitive (3D-7) and -resistant (RKL-2) strains of $P$. falciparum, which, however, was considerably less than that of the standard reference drug, CQ.

\section{About Authors}

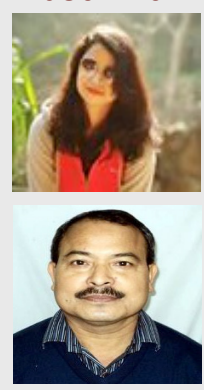

Ms. Arundhati Medhi: Has completed M. Pharm. with specialization in Pharmaceutical Chemistry from Dept. of Pharmaceutical Sciences, Dibrugarh University, Dibrugarh, Assam (India).

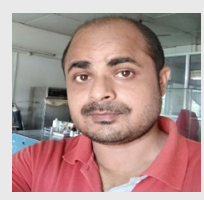

Dr. Dipak Chetia: Is Professor and Head at the Dept. of Pharmaceutical Sciences, Dibrugarh University, Dibrugarh, Assam (India). Dr. Chetia has been engaged in teaching and research since 1990. His research interests include antimalarial drug development from natural and synthetic sources. He has published a good number of papers in various journals and books.

Mr. Mithun Rudrapal: Is currently pursuing his Ph. D. degree in antimalarial drug discovery and development at the Dept. of Pharmaceutical Sciences, Dibrugarh University, Dibrugarh, Assam (India). Mr. Rudrapal completed M. Pharm. with specialization in Pharm. Chemistry from the same University. He has published number of papers in various journals of national and international repute.

Cite this article: Arundhati M, Dipak C, Mithun R. Synthesis and Antimalarial Activity of Lawsone Mannich Base Derivatives. Indian J of Pharmaceutical Education and Research. 2018;52(3):472-9. 